\title{
Development and validation of a nomogram model for early postoperative hyperlactatemia in patients undergoing cardiac surgery
}

\author{
Su Wang ${ }^{1 \#}$, Dashuai Wang ${ }^{2 \#}$, Xiaofan Huang ${ }^{2}$, Hongfei Wang ${ }^{2}$, Sheng Le ${ }^{2}$, Jinnong Zhang ${ }^{1}$, Xinling Du ${ }^{2}$ \\ ${ }^{1}$ Department of Emergency Medicine, Union Hospital, Tongji Medical College, Huazhong University of Science and Technology, Wuhan, China; \\ ${ }^{2}$ Department of Cardiovascular Surgery, Union Hospital, Tongji Medical College, Huazhong University of Science and Technology, Wuhan, China \\ Contributions: (I) Conception and design: X Du, J Zhang, X Huang, S Wang, D Wang; (II) Administrative support: X Du, J Zhang, H Wang, S Le; (III) \\ Provision of study materials or patients: X Du, X Huang, H Wang; (IV) Collection and assembly of data: D Wang, H Wang; (V) Data analysis and \\ interpretation: S Wang, D Wang, S Le, X Huang; (VI) Manuscript writing: All authors; (VII) Final approval of manuscript: All authors. \\ \#These authors contributed equally to this work. \\ Correspondence to: Xiaofan Huang, MD, Union Hospital, Wuhan Jiefang Road, No. 1277, Wuhan 430022, China. Email: dr_xfhuang@hust.edu.cn; \\ Xinling Du, MD, PhD. Union Hospital, Wuhan Jiefang Road, No. 1277, Wuhan 430022, China. Email: xinlingdu@hust.edu.cn; Jinnong Zhang, \\ MD, PhD. Union Hospital, 1277 Jiefang Avenue, Wuhan 430022, China. Email: zhangjnwhhb@163.com.
}

\begin{abstract}
Background: Postoperative hyperlactatemia (POHL) is common in patients undergoing cardiac surgery, associated with adverse outcomes. The aim of this study was to identify predictors for POHL after cardiac surgery and to develop and validate a predictive model.

Methods: Adult patients who underwent open heart surgery at our institution between 2016 and 2019 were retrospectively included. The patients were randomly divided into training and validation groups at a 2:1 ratio. Multivariate logistic regression was performed to identify independent predictors for POHL in the training set. A nomogram was then constructed and was validated in the validation set.

Results: POHL developed in 713 of the 5,323 patients (13.4\%). The mortality rate was higher in patients with POHL compared with patients without that $(9.5 \%$ vs. $2.1 \%, \mathrm{P}<0.001)$. Age, white blood cell (WBC) count, left ventricular ejection fraction, renal insufficiency, cardiac surgery history, red blood cell (RBC) transfusion, and cardiopulmonary bypass (CPB) time were identified as independent risk factors. The nomogram based on these predictors indicated good discrimination in both the training (c-index: 0.787) and validation (c-index: 0.820 ) sets. The calibration was reasonable by both visual inspection and goodness-of-fit test. The decision and clinical impact curves demonstrated good clinical utility.

Conclusions: We identified 7 independent risk factors and derived a prediction model for POHL in patients undergoing cardiac surgery. The model may contribute significantly to early risk assessment and clinical intervention.
\end{abstract}

Keywords: Postoperative hyperlactatemia (POHL); cardiac surgery; risk factor; prediction model; nomogram

Submitted Jun 18, 2021. Accepted for publication Aug 13, 2021.

doi: $10.21037 /$ jtd-21-1004

View this article at: https://dx.doi.org/10.21037/jtd-21-1004

\section{Introduction}

Hyperlactatemia is a common complication in critically ill patients and is a strong indicator for poor prognosis (1). The development of hyperlactatemia in intensive care unit (ICU) is closely associated with increased mortality among different critically ill patients including those undergoing cardiac surgery (2). The association between postoperative hyperlactatemia (POHL) and various adverse outcomes has been widely reported, such as cardiogenic shock, acute renal failure, acute respiratory distress syndrome, and mortality 
(3-6). The incidence of POHL differed considerably in the literature, with reported rates of 20.6-71.8\% $(4,5,7)$.

The bimodal distribution of POHL has been seen in patients undergoing cardiac surgery. Early-onset hyperlactatemia occurs during or soon after cardiopulmonary bypass $(\mathrm{CPB})$ and late-onset hyperlactatemia develops within the first 12 hours after ICU admission (8). Some studies focused on identifying independent risk factors for POHL after cardiac surgery have been conducted and several predictors have been reported, such as elevated level of preoperative serum creatinine, longer $\mathrm{CPB}$ duration, higher blood glucose and reduced oxygen delivery $(9,10)$. However, the physiological and clinical data suggested that lateonset hyperlactatemia was not attributed to impaired tissue perfusion or oxygen delivery, and the influencing factors may vary with time (11). Furthermore, although several risk factors have been described in the literature $(4,12,13)$, to our knowledge, there are no existing nomogram models to predict the occurrence of POHL in patients undergoing cardiac surgery. The construction of a specific and sensitive nomogram model for POHL after cardiac surgery is still urgently needed.

The aims of this study were to identify preoperative and intraoperative predictors for POHL in patients undergoing cardiac surgery, and to establish and validate a nomogram model to help with risk evaluation and clinical prevention.

We present the following article in accordance with the TRIPOD reporting checklist (available at https://dx.doi. org/10.21037/jtd-21-1004).

\section{Methods}

\section{Ethical statement}

This study was approved by the Medical Ethics Committee of Tongii Medical College, Huazhong University of Science and Technology (IORG No. IORG0003571) and was conducted in accordance with the Declaration of Helsinki (as revised in 2013). Written informed consent was waived due to the retrospective, observational nature.

\section{Patient selection}

We included consecutive adult patients undergoing open heart surgery from January 2016 to December 2019 in a tertiary care center with more than 6,000 beds, located in Wuhan, China. All patients undergoing cardiac surgery were admitted to the ICU postoperatively and received mechanical ventilatory support. The exclusion criteria were set as follows: intraoperative death, immunosuppression or immune deficiency, organ transplantation, and incomplete data recording.

\section{Data collection}

We obtained clinical data through electronic medical record system of our institution. Demographics included age, gender, calculated body mass index, history of smoking and drinking. Comorbidities contained hypertension, diabetes mellitus, cerebrovascular disease, pericardial effusion, left ventricular ejection fraction (LVEF), atrial fibrillation, pulmonary artery hypertension, chronic obstructive pulmonary disease, gastrointestinal tract disease, renal insufficiency, peripheral vascular disease, cardiac and general surgical history. Laboratory variables included red blood cell (RBC) count, hemoglobin, white blood cell (WBC) count, platelet count, serum creatinine, globulin, albumin, total bilirubin, direct bilirubin, alanine aminotransferase, aspartate aminotransferase, and gamma-glutamyl transpeptidase. Operative variables included surgical types, blood transfusion, $\mathrm{CPB}$ and aortic cross clamp time. Postoperative data included postoperative pneumonia, readmission to ICU, reintubation, tracheotomy, intra-aortic balloon pump, continuous renal replacement therapy, mortality, and the lengths of mechanical ventilation, ICU and hospital stay.

\section{Measurement and definition}

The concentration of arterial lactate was measured using a blood gas analyzer (Radiometer, type ABL80PLEX, Denmark). The recorded value represented the peak blood lactate value in the first 12 hours postoperatively. In this study, POHL was defined as arterial blood lactate level exceeding $4 \mathrm{mmol} / \mathrm{L}$ with reference to previous studies $(7,14)$.

Body mass index was calculated as weight $(\mathrm{kg}) /$ height $\left(\mathrm{m}^{2}\right)$. Smoking history was defined as current or previous daily smoking. Drinking history was defined as having a history of habitual drinking. Hypertension was defined as blood pressure $\geq 140 / 90 \mathrm{mmHg}$, medication for hypertension, or a history of hypertension. Diabetes mellitus was defined as fasting glucose $\geq 7.0 \mathrm{mmol} / \mathrm{L}$, non-fasting glucose $\geq 11.1 \mathrm{mmol} / \mathrm{L}$, medication for diabetes, or self-reported diabetes mellitus. Chronic obstructive pulmonary disease was defined as FEV1/FVC $\leq 0.7$. Renal insufficiency was defined as serum creatinine $>110 \mu \mathrm{mol} / \mathrm{L}$ or previous diagnosis of renal insufficiency. 


\section{Statistical analysis}

Statistical analyses were performed using $\mathrm{R}$ software (version 4.0.3). A two-tailed $\mathrm{P}$ value $<0.05$ was considered statistically significant. Categorical variables were expressed as frequency (percentage) and analyzed by the chi-square test or Fisher's exact test. Normally distributed continuous variables were presented as means \pm standard deviations and analyzed by Student's $t$-test. Non-normally distributed variables were expressed as medians with inter-quartile ranges and analyzed by Mann-Whitney $U$ test. The complete datasets were randomly divided into training and validation sets by $2: 1$ ratio. The former was applied for model establishment, and the latter was applied for model validation. In the training set, the preoperative and intraoperative variables were first analyzed by univariate analysis to screen possible risk factors for POHL. Variables with $\mathrm{P}$ value $<0.1$ were then included in multivariate logistic regression analysis to identify independent risk factors.

Based on the multivariate model, a nomogram was constructed to facilitate a better prediction of POHL after cardiac surgery. Internal validation of the nomogram model was evaluated by bootstrapping using 1,000 replications. External validation was conducted in the independent validation set. One smoothed non-parametric calibration curve and one fitted logistic calibration curve were used to visually assess the calibration. The Hosmer-Lemeshow goodness-of-fit statistic was also calculated. The area under the receiver operating characteristic (ROC) curve (AUC) or c-index was employed to evaluate the discrimination. Comparison of the AUC between two sets was performed using the Delong method (15). Decision curve analysis was utilized to evaluate the clinical utility of the model. The decision curve exhibited standardized net benefit against threshold probability. The clinical impact curve displayed the number of judged high risk with different threshold probability and the number of true positives among 1,000 patients.

\section{Results}

\section{Baseline demographics and clinical characteristics}

Among the 5,784 adult patients who underwent cardiac surgery, 5 died intraoperatively, 375 had immunosuppression, immune deficiency or organ transplantation, and 81 had missing data (Figure 1). The remaining 5,323 cases fulfilled the inclusion criteria and were further analyzed (Table 1). The average age of the included patients was 51.2 years, and $56.4 \%$ were males. The overall incidence of POHL was $13.4 \%$.

Of the 5,323 operations, isolated valve replacement or repair was performed for 2,897 cases $(54.4 \%)$, isolated coronary artery bypass grafting for 591 cases $(11.1 \%)$, mixed valve surgery and coronary artery bypass grafting for 481 cases $(9.0 \%)$, aortic surgery for 904 cases $(17.0 \%)$, and other types for 450 cases (8.5\%).

The study population had different comorbidities. Patients with smoking history made up $29.1 \%$, drinking history $21.7 \%$, diabetes mellitus $8.2 \%$, hypertension $30.1 \%$, atrial fibrillation $17.1 \%$, chronic obstructive pulmonary disease $10.5 \%$, gastrointestinal tract disease $8.4 \%$, renal insufficiency $9.8 \%$, cardiac surgery history $7.1 \%$. The average duration of $\mathrm{CPB}$ was 105 minutes, and the median volume of intraoperative transfusion of RBC was 1 unit. The baseline characteristics, comorbidities and operative variables were similar in the training and validation sets (Table 1). The incidence of POHL in the two sets was $13.1 \%$ and $14.0 \%$, respectively $(\mathrm{P}=0.38)$.

\section{Development of the nomogram model}

Univariate analysis of possible predictors for POHL after cardiac surgery in the training set was displayed in Table 2. Before the construction of a multivariate model, collinearity diagnostics were performed. Significant risk factors in the univariate analysis were further entered into the multivariate logistic regression analysis. Seven independent predictors associated with the development of POHL were identified, including renal insufficiency, cardiac surgery history, WBC count, age, LVEF, intraoperative transfusion of RBC, and CPB time (Table 3). A nomogram used to predict the risk of POHL after cardiac surgery was established based on the multivariate logistic regression model (Figure 2A). Regression coefficients of the variables were scaled to scores within the range of $0-100$, reflecting their relative importance.

The probability of POHL after cardiac surgery in a specific patient can be calculated easily by summing the scores of all associated factors. Older patients who had renal insufficiency, previous cardiac surgery, higher WBC count, lower LVEF, more intraoperative transfusion of RBC, and longer duration of CPB had higher scores and thus higher risk of POHL after cardiac surgery.

\section{Assessment and validation of the nomogram model}

Both internal and external validation were used to validate 


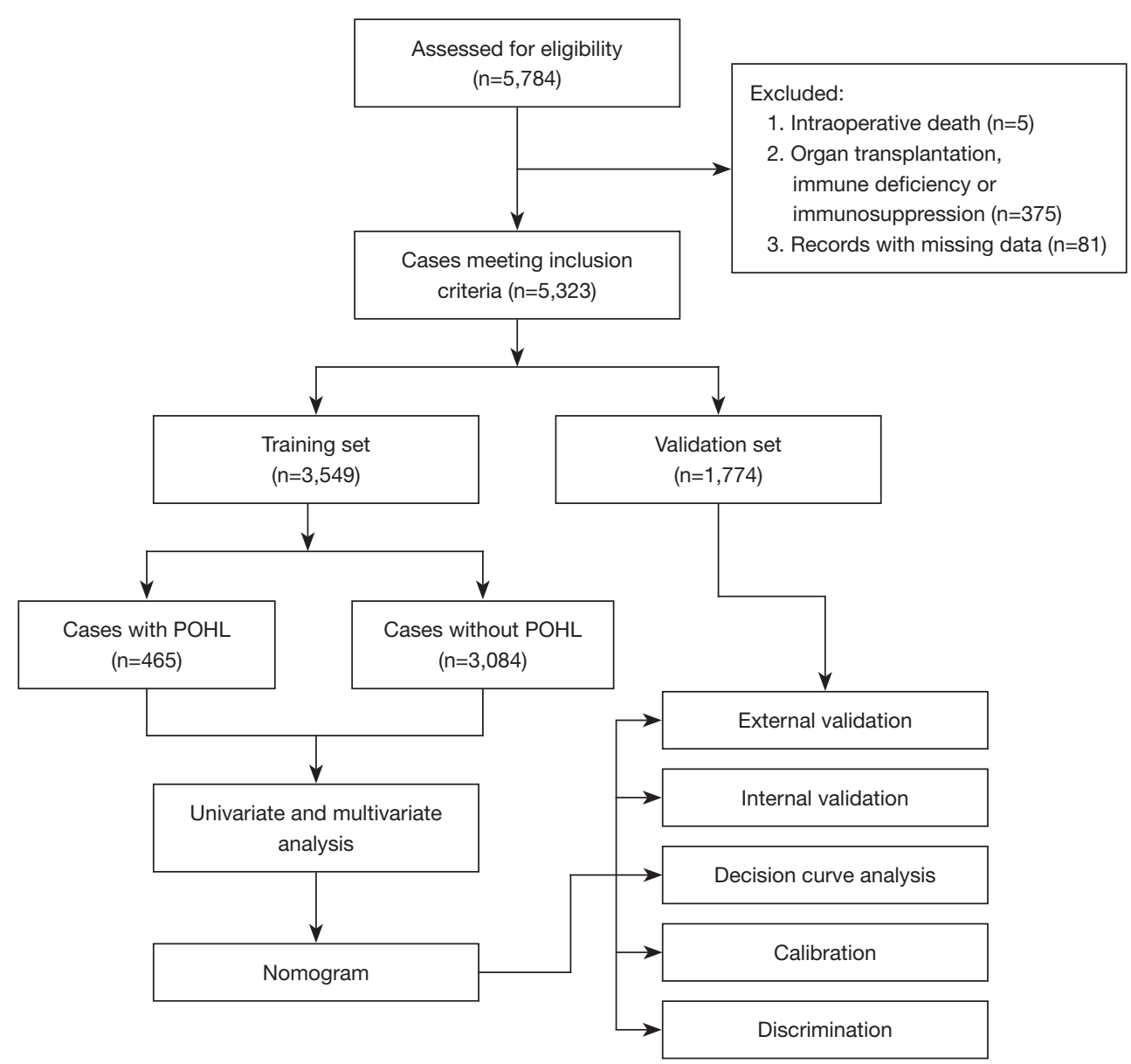

Figure 1 Flow chart of the study. POHL, postoperative hyperlactatemia.

Table 1 Comparison of characteristics between the training and validation sets

\begin{tabular}{|c|c|c|c|}
\hline Characteristic & Training set, $\mathrm{n}=3,549(\%)$ & Validation set, $\mathrm{n}=1,774$ (\%) & $P$ value \\
\hline \multicolumn{4}{|l|}{ Demographics } \\
\hline Male & $1,982(55.8)$ & $1,019(57.4)$ & 0.269 \\
\hline Age (years) & $51.34 \pm 12.81$ & $50.85 \pm 13.12$ & 0.196 \\
\hline Body mass index $\left(\mathrm{kg} / \mathrm{m}^{2}\right)$ & $23.1(20.9,25.5)$ & $23.2(20.9,25.6)$ & 0.806 \\
\hline Drinking history & $769(21.7)$ & $385(21.7)$ & 0.977 \\
\hline \multicolumn{4}{|l|}{ Underlying conditions } \\
\hline Hypertension & $1,073(30.2)$ & $531(29.9)$ & 0.821 \\
\hline Diabetes mellitus & $289(8.1)$ & $149(8.4)$ & 0.749 \\
\hline
\end{tabular}

Table 1 (continued) 
Table 1 (continued)

\begin{tabular}{|c|c|c|c|}
\hline Characteristic & Training set, $n=3,549(\%)$ & Validation set, $\mathrm{n}=1,774$ (\%) & $P$ value \\
\hline Gastrointestinal tract disease & $300(8.5)$ & $147(8.3)$ & 0.836 \\
\hline Atrial fibrillation & $600(16.9)$ & $312(17.6)$ & 0.534 \\
\hline General surgery history & $997(28.1)$ & $500(28.2)$ & 0.944 \\
\hline Pulmonary artery hypertension & $915(25.8)$ & $475(26.8)$ & 0.437 \\
\hline Pericardial effusion & $504(14.2)$ & $252(14.2)$ & 0.997 \\
\hline Left ventricular ejection fraction (\%) & $62[58,66]$ & $62[58,67]$ & 0.270 \\
\hline \multicolumn{4}{|l|}{ Laboratory values } \\
\hline Hemoglobin (g/L) & $129(118,140)$ & $130(118,141)$ & 0.119 \\
\hline Platelet count $\left(\times 10^{9} / \mathrm{L}\right)$ & $180(144,221)$ & $179(145,221)$ & 0.738 \\
\hline Serum creatinine $(\mu \mathrm{mol} / \mathrm{L})$ & $71.9(60.9,85.3)$ & $72.1(60.9,85.3)$ & 0.613 \\
\hline Serum albumin (g/L) & $40.4(37.9,42.6)$ & $40.4(38.0,42.8)$ & 0.341 \\
\hline Serum globulin (g/L) & $24.3(21.6,27.3)$ & $24.5(21.8,27.3)$ & 0.253 \\
\hline Total bilirubin $(\mu \mathrm{mol} / \mathrm{L})$ & $13.5(10.3,18.6)$ & $13.3(10.3,18.3)$ & 0.585 \\
\hline Direct bilirubin $(\mu \mathrm{mol} / \mathrm{L})$ & $4.1(3.4,6.2)$ & $4.3(3.5,6.3)$ & 0.128 \\
\hline Alanine aminotransferase (U/L) & $20.4(17.3,30.7)$ & $20.5(17.5,40.0)$ & 0.509 \\
\hline Mixed valve and coronary surgery & $310(8.7)$ & $171(9.6)$ & 0.278 \\
\hline Aortic surgery & $604(17.0)$ & $300(16.9)$ & 0.921 \\
\hline Other types & $300(8.5)$ & $150(8.5)$ & 0.998 \\
\hline Emergent cardiac surgery & $335(9.4)$ & $157(8.9)$ & 0.484 \\
\hline Cardiopulmonary bypass time (minutes) & $105[78,142]$ & $106[79,142]$ & 0.556 \\
\hline Aortic cross clamp time (minutes) & $69[46,96]$ & $69[47,94]$ & 0.897 \\
\hline Transfusion of red blood cells (units) & $1[1,3]$ & $1[1,3]$ & 0.639 \\
\hline
\end{tabular}


Table 2 Univariate analysis of possible risk factors for POHL after cardiac surgery in the training set

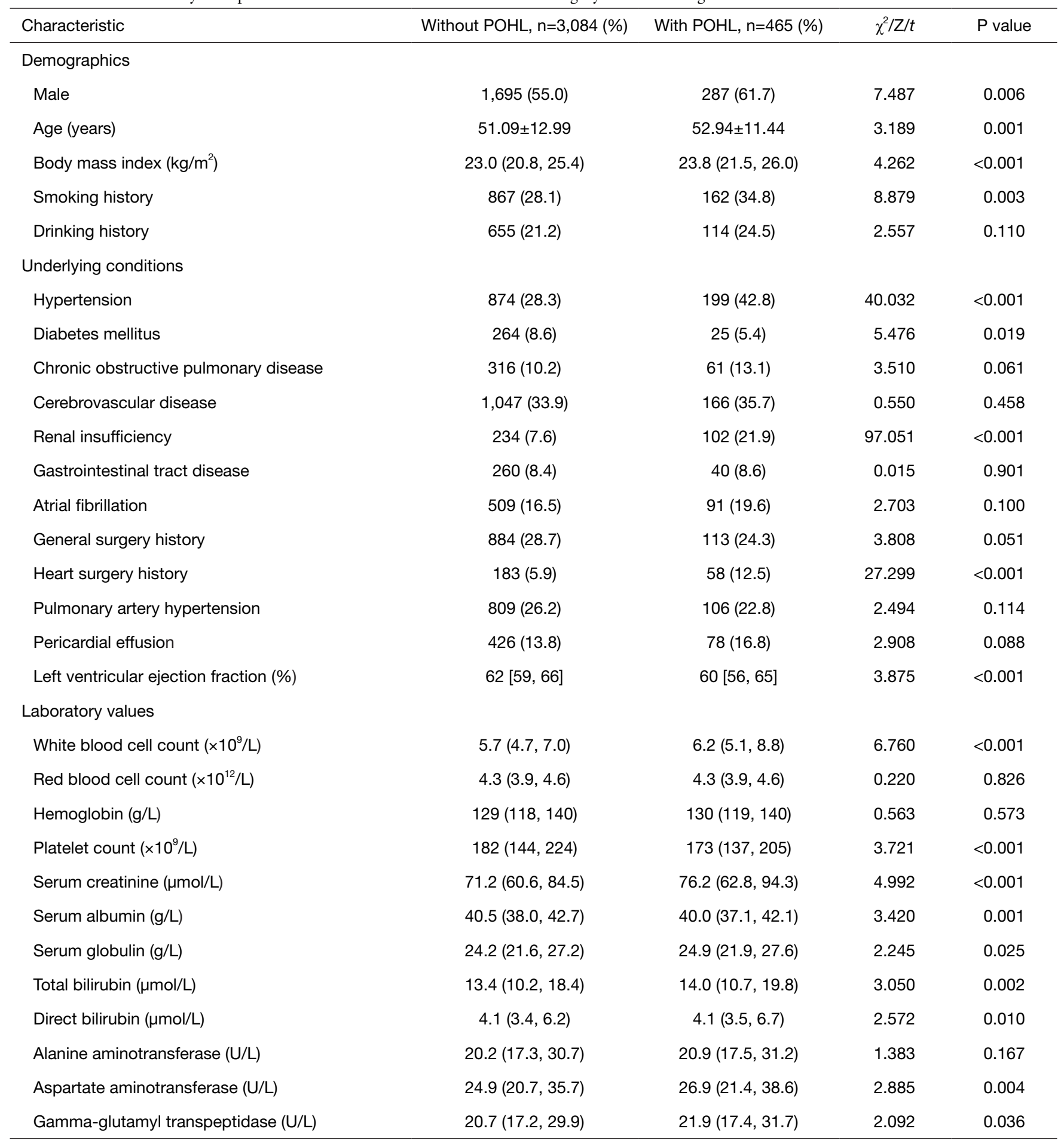

Table 2 (continued) 
Table 2 (continued)

\begin{tabular}{|c|c|c|c|c|}
\hline Characteristic & Without POHL, $n=3,084(\%)$ & With POHL, $\mathrm{n}=465$ (\%) & $\chi^{2} / Z / t$ & $P$ value \\
\hline \multicolumn{5}{|l|}{ Operative variables } \\
\hline Surgical types & & & 192.822 & $<0.001$ \\
\hline Isolated valve surgery & $1,740(56.4)$ & $180(38.7)$ & & \\
\hline Isolated coronary artery bypass grafting & $381(12.4)$ & $34(7.3)$ & & \\
\hline Aortic surgery & $439(14.2)$ & $165(35.5)$ & & \\
\hline Other types & $288(9.3)$ & $12(2.6)$ & & \\
\hline Emergent cardiac surgery & $208(6.7)$ & $127(27.3)$ & 199.958 & $<0.001$ \\
\hline Cardiopulmonary bypass time (minutes) & $100[75,132]$ & $157[114,220]$ & 18.328 & $<0.001$ \\
\hline
\end{tabular}

POHL, postoperative hyperlactatemia.

Table 3 Multivariate analysis of independent risk factors for POHL after cardiac surgery

\begin{tabular}{|c|c|c|c|c|}
\hline Characteristic & Coefficient & Standard error & OR $(95 \% \mathrm{Cl})$ & $P$ value \\
\hline Cardiac surgery history & 0.678 & 0.181 & $1.970(1.383-2.806)$ & $<0.001$ \\
\hline WBC count $\left(\times 10^{9} / L\right)$ & 0.046 & 0.019 & $1.047(1.008-1.088)$ & 0.017 \\
\hline Age (years) & 0.010 & 0.005 & $1.010(1.001-1.019)$ & 0.038 \\
\hline Transfusion of RBC (units) & 0.161 & 0.024 & 1.175 (1.120-1.233) & $<0.001$ \\
\hline CPB time (minutes) & 0.767 & 0.072 & $2.152(1.867-2.481)$ & $<0.001$ \\
\hline Intercept & -3.607 & 0.534 & 0.027 & $<0.001$ \\
\hline
\end{tabular}

$\mathrm{Cl}$, confidence interval; CPB, cardiopulmonary bypass; LVEF, left ventricular ejection fraction; OR, odds ratio; POHL, postoperative hyperlactatemia; RBC, red blood cell; WBC, white blood cell.

the nomogram model. One thousand bootstrap resamples were used for internal validation in the training set. External validation was performed in the independent validation set. To evaluate the predictive capability of the nomogram, the ROC curves were drawn (Figure 2B). The AUCs in the training and validation sets were respectively 0.79 (95\% CI, $0.77-0.81$ ) and 0.82 (95\% CI, 0.79-0.85), both indicating reasonable discrimination. To assess the calibration of the nomogram model, Hosmer-Lemeshow goodness-of-fit test was used, and calibration curves were plotted. The model was well calibrated in both the training (Hosmer-Lemeshow $\left.\chi^{2}=11.88, \mathrm{P}=0.16\right)$ and validation sets (Hosmer-Lemeshow $\left.\chi^{2}=9.94, \mathrm{P}=0.27\right)$. By visual inspection, the nomogram also showed good calibration in the two sets (Figure 2C,2D).

\section{Clinical utility of the nomogram model}

To evaluate the clinical utility of the prediction model, decision curve analysis was performed, as well as graphical decision and clinical impact curves. The decision curves of the model demonstrated that compared with "intervention for all" or "no intervention" strategies, more clinical net benefits could be obtained using the nomogram model when the risk threshold was between 0.02 and 0.60 (Figure 3A,3B). 
A
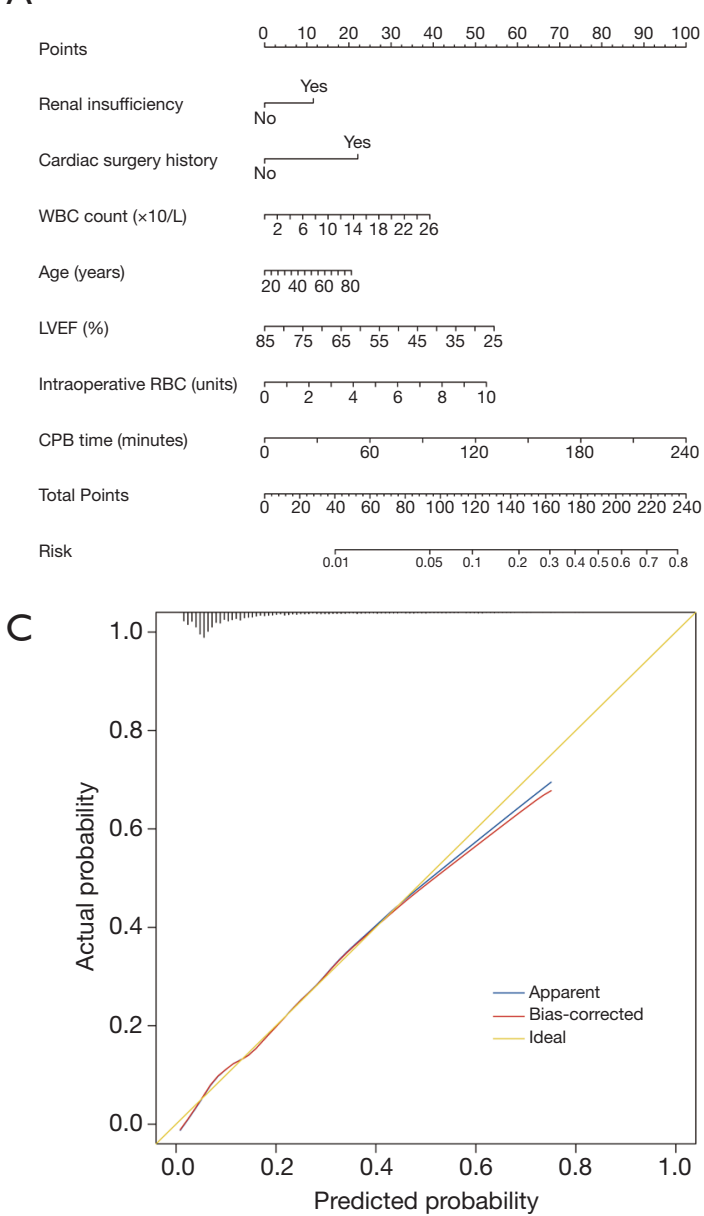
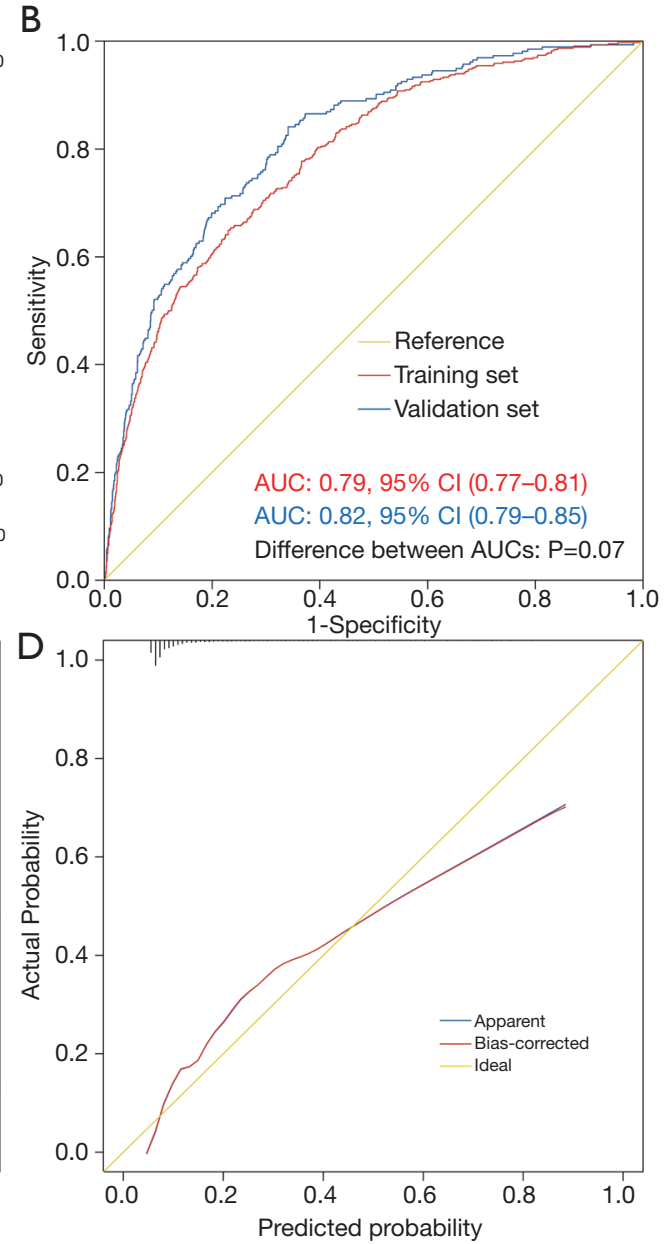

Figure 2 Development, assessment and validation of the nomogram model. (A) Nomogram predicting the development of postoperative hyperlactatemia in patients undergoing cardiac surgery; (B) ROC curves for the nomogram in the training and validation sets; (C) calibration plot of the nomogram in the training set; and (D) calibration plot of the nomogram in the validation set. AUC, area under the receiver operating characteristic curve; CI, confidence interval; CPB, cardiopulmonary bypass; LVEF, left ventricular ejection fraction; RBC, red blood cell; ROC, receiver operating characteristic; WBC, white blood cell.

The clinical impact curves also illustrated that the nomogram model had remarkable predictive power and exhibited good clinical utility (Figure 3C,3D).

\section{Risk stratification}

According to the nomogram model, we defined four risk groups as very low risk ( $<94$ points), low risk (94-118 points), medium risk (119-162 points), and high risk ( $>162$ points) for POHL after cardiac surgery (Table 4). The corresponding cutoff values of the estimated probabilities was $0.05,0.1$, and 0.3 . In this study, about $30 \%$ of the patients were respectively divided into very low-, low-, and medium-risk groups, and about $10 \%$ into high-risk group. The difference of probabilities between each risk interval was significant $(\mathrm{P}<0.05)$ and the model-based risk stratification performed well.

\section{Outcomes}

POHL developed in 713 of the 5,323 patients (13.4\%). The overall mortality rate was $3.1 \%$, with a rate of $9.5 \%$ in patients with POHL versus $2.1 \%$ in those without POHL $(\mathrm{P}<0.001)$. Furthermore, POHL was associated with a significantly higher rate of intra-aortic balloon pump requirement, continuous renal replacement therapy requirement, postoperative pneumonia, readmission to 

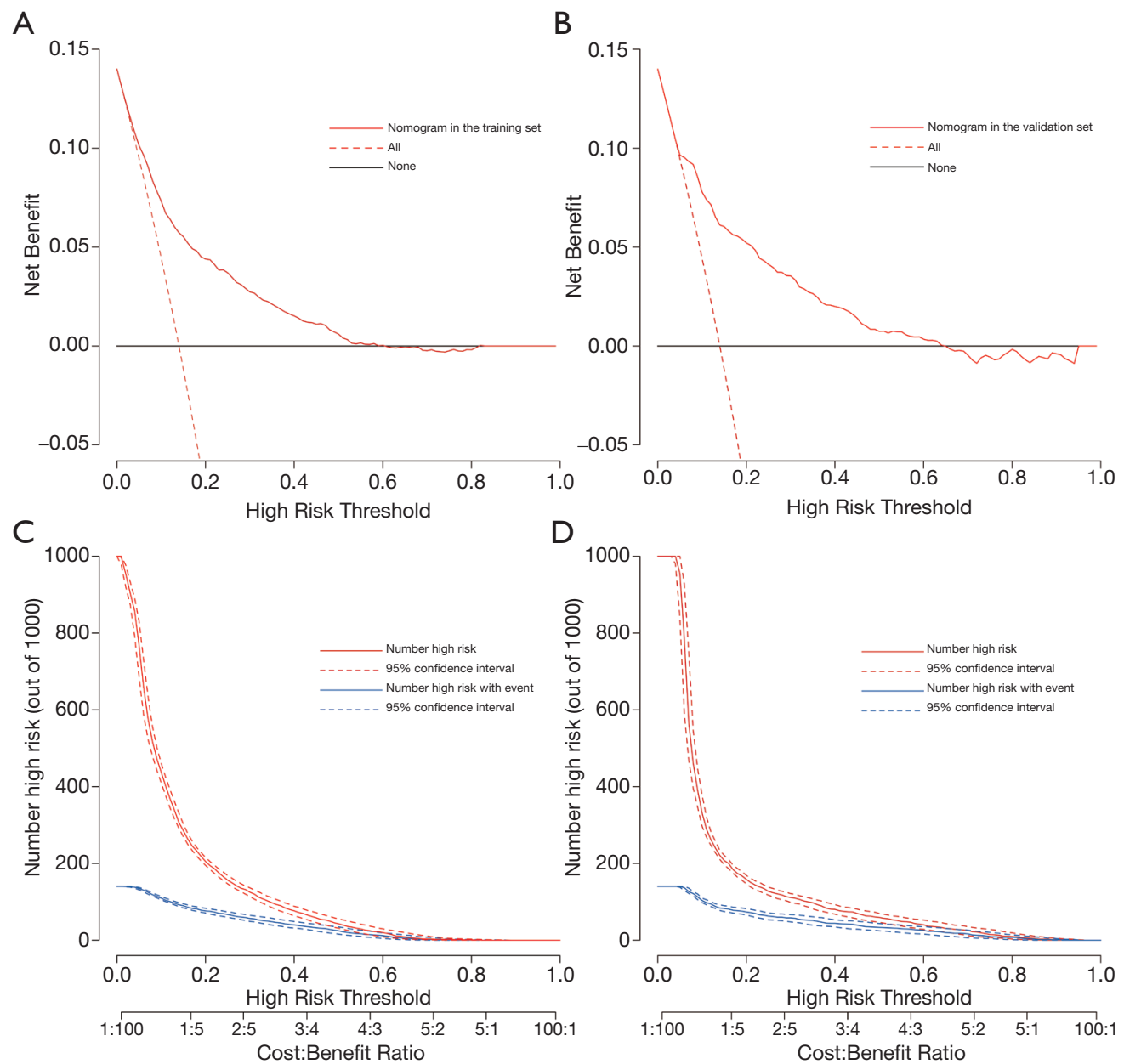

Figure 3 Decision curve analysis of the nomogram model. Decision curves of the nomogram in the training (A) and validation sets (B), and clinical impact curves of the nomogram in the training $(C)$ and validation sets (D).

Table 4 Risk intervals of POHL based on the nomogram model

\begin{tabular}{lcccc}
\hline Risk intervals & $\begin{array}{c}\text { Very low-risk } \\
(<94 \text { points })\end{array}$ & $\begin{array}{c}\text { Low-risk } \\
(94-118 \text { points })\end{array}$ & $\begin{array}{c}\text { Medium-risk } \\
(119-162 \text { points })\end{array}$ & $\begin{array}{c}\text { High-risk } \\
(>162 \text { points })\end{array}$ \\
\hline Estimated probability (\%) & $<5$ & $5-10$ & $10-30$ & $17.2(15.3-19.0)$ \\
Observed probability, \% (95\% Cl) & $1.9(1.2-2.7)$ & $7.6(6.3-8.9)$ & $1,577(29.6)$ & $57.4(43.4-51.4)$ \\
No. of patients (\%) & $1,438(27.0)$ & $1,709(32.1)$ & & $599(11.3)$
\end{tabular}

$\mathrm{Cl}$, confidence interval; $\mathrm{POHL}$, postoperative hyperlactatemia.

ICU, reintubation, and tracheotomy. The postoperative lengths of mechanical ventilation, ICU and hospital stay also extended significantly in patients with POHL. Details of comparison between patients with and without POHL after cardiac surgery were presented in Table 5 .

\section{Discussion}

The level of blood lactate is known to be closely related to the prognosis of critically ill patients and hyperlactatemia is strongly associated with higher mortality (16), which is 
Table 5 Postoperative variables in patients with and without POHL after cardiac surgery

\begin{tabular}{|c|c|c|c|c|c|}
\hline Variables & $\begin{array}{l}\text { All patients, } \\
n=5,323(\%)\end{array}$ & $\begin{array}{c}\text { Without POHL, } \\
n=4,610(\%)\end{array}$ & $\begin{array}{c}\text { With POHL, } \\
n=713(\%)\end{array}$ & $\chi^{2} / Z$ & $P$ value \\
\hline Postoperative pneumonia & $530(10.0)$ & $341(7.4)$ & $189(26.5)$ & 251.547 & $<0.001$ \\
\hline Reintubation & $247(4.6)$ & $171(3.7)$ & $76(10.7)$ & 67.403 & $<0.001$ \\
\hline Tracheotomy & $128(2.4)$ & $75(1.6)$ & $53(7.4)$ & 88.711 & $<0.001$ \\
\hline Mechanical ventilation (hours) & $23[18,44]$ & $22[18,40]$ & $45[25,91]$ & 21.834 & $<0.001$ \\
\hline Readmission to ICU & $207(3.9)$ & $151(3.3)$ & $56(7.9)$ & 34.635 & $<0.001$ \\
\hline ICU stay (days) & $3[2,5]$ & $3[2,4]$ & $5[4,9]$ & 22.621 & $<0.001$ \\
\hline
\end{tabular}

POHL, postoperative hyperlactatemia; ICU, intensive care unit.

reconfirmed by our results. The incidence of POHL was $13.4 \%$ in this study, similar to previous reports $(14,17)$. The overall mortality was $3.1 \%$, but it increased significantly in patients with POHL. In addition, more frequent complications and longer hospital stays were observed in these patients, consistent with results in the literature $(5,7,17)$. The poorer outcomes of patients with POHL emphasized the need of identifying risk factors and highrisk population.

Although some studies focused on POHL have been conducted, to our knowledge, this is the first large-sized study aiming to develop and validate a nomogram model for POHL after cardiac surgery. In this study, we used clinical data of 5,323 patients to develop and validate a risk prediction model for POHL after cardiac surgery. Five preoperative and two intraoperative independent predictors were identified. The nomogram indicated good predictive power and was well calibrated. Decision curve analysis of the nomogram demonstrated good clinical utility. Four risk intervals were identified as very low-, low-, medium- and high-risk to facilitate clinical practice.

The duration of CPB was identified as a significant predictor for POHL after cardiac surgery in our analysis, which was in agreement with previous studies $(4,18)$. The association between $\mathrm{CPB}$ duration and early-onset or lateonset hyperlactatemia has been verified by some published studies, however, some other studies yielded different results (19-21). The occurrence of POHL after cardiac surgery may be caused by a mixture of early- and late-onset hyperlactatemia mechanisms and the change of the lactate levels was related to the physiopathologic mechanisms and the efficacy of the treatment (22). Lactate-guided therapy on patients with POHL at ICU arrival can significantly decrease the hospital mortality, and intervention at an early stage (before or during surgery) may be more valuable to reduce risk (23).

The specific relationship between $\mathrm{CPB}$ and POHL may be complex. Tissue micro-dialysis studies indicated that CPB itself can cause elevated lactate level and increased lactate-pyruvate ratio in the myocardial and non-myocardial tissue $(24,25)$. One prospective study observed that pulmonary lactate release following $\mathrm{CPB}$ could continue up to six hours, which meant that the washout of lactate from previous hypoperfused tissues could partly account for the development of POHL (26). In addition, the process of $\mathrm{CPB}$ exposes patients to the complex non-physiological conditions. The contact of blood components with artificial surfaces of $\mathrm{CPB}$ circuit could trigger inflammatory response, resulting in lactate accumulation $(27,28)$. Miniaturized CPB circuit has been reported to be able to diminish the inflammatory response as a consequence of less exposure, and its application may be conducive to reducing the risk of POHL (29). Moreover, CPB may damage peripheral perfusion and metabolic balance due to capillary collapse and stasis, and the degree of this damage may significantly increase over time (30). Longer duration of $\mathrm{CPB}$ is generally accompanied by longer duration of surgery, anesthesia, and aortic cross-clamping. As a 
modifiable factor, minimizing the $\mathrm{CPB}$ duration when allowed may yield a better prognosis.

Intraoperative transfusion of RBC was another independent risk factor in our analysis. Although the intended therapeutic effect of blood transfusion in cardiac surgery was to improve organ function and save life, increasing evidence indicated that transfused RBCs might actually affect tissue oxygenation and contribute to organ injury $(31,32)$. Several studies have found that the risk of adverse outcomes may gradually increase with each more unit of RBCs transfused $(33,34)$, which was consistent with our results. RBC transfusion could cause proinflammatory and immunosuppressive effects, and stored RBCs might be less deformable and supple, causing micro-circulatory occlusion and thus organ ischemia $(35,36)$. Furthermore, studies revealed that decreased $\mathrm{RBC}$ deformability was closely associated with elevated RBC distribution width, which had a positive correlation with POHL and could even predict multiple organ dysfunction syndrome $(37,38)$. Retrograde autologous priming has recently been reported to be an effective adjunct to reduce transfusion requirements and limit haemodilution (39). A meta-analysis also demonstrated that restrictive transfusion was safe, and a threshold of hemoglobin concentration $<7.5 \mathrm{~g} / \mathrm{dL}$ was recommended for patients undergoing cardiac surgery $(40,41)$.

WBC count was found to be independently associated with the development of POHL after cardiac surgery in our analysis, however, none of the published studies reported this. It is known that leukocytosis represents a proinflammatory state. Pre-existing activation of the leukocytes could impair the perfusion at microcirculatory level after CPB onset (42). Increased preoperative WBC was also found to be significantly associated with increased need for intra-aortic balloon pump, which may indirectly associate with insufficient perfusion (43). The role of leukodepletion in cardiac surgery remains unclear, however, delaying surgery to reduce the elevated $\mathrm{WBC}$ to normal levels when possible may help prevent POHL (44-46).

Lower LVEF, renal insufficiency, previous cardiac surgery, and advanced age were also identified as independent risk factors for POHL after cardiac surgery in this study, which was also reported in previous studies $(5,13,47)$. Lazzeri et al. found a significant relationship between LVEF and lactate values and reported that hemodynamic impairment was possibly responsible for hyperlactatemia (48). Mustafa et al. indicated that the CPB procedure decreased lactate clearance, which may be related to liver dysfunction (49). Although metabolic clearance is mainly hepatic, studies have found that the kidney also plays an important role in the clearance of lactate (50). A significant reduced lactate clearance was possibly observed in patients with renal insufficiency and hypoperfusion, leading to the development of hyperlactatemia. Zymliński et al. found that patients without clinical signs of peripheral hypoperfusion might also have an elevated lactate level, which was also related to worse prognosis (51).

Some variables such as hyperglycemia and epinephrine were identified as predictors for POHL in published studies, but were not in our analysis. The role of hyperglycemia in predicting hyperlactatemia has been highlighted by Ranucci et al., and they deemed that the stress response of cardiac surgery caused this result (9). Epinephrine is always used to treat postoperative low cardiac output syndrome, whereas it may increase the incidence of POHL (4). The increased glycogenolysis may be responsible for the elevated lactate levels in patients with epinephrine administration (20). However, the postoperative cardiac output and epinephrine use were not included in our analysis due to their nature of postoperative variables and cannot be obtained early.

This prediction model may play an important role in identifying high-risk POHL populations and have clinical utility in reducing the occurrence of POHL. Compared to conventional therapies, risk factors-oriented and nomogram-based early interventions may significantly improve outcomes.

Some limitations should be mentioned. First, this study was conducted in a single cardiac center, which may restrict the wide application of the model to other centers. External validation in other centers may be required to further verify its clinical effectiveness. Second, it is undeniable that intraoperative and postoperative hemodynamic status and medication use may affect lactate metabolism, however, these variables were not included in our analysis. This is partly because that a large proportion of these variables are missing or have no exact records, which is mainly limited by the retrospective nature of the data collection. More appropriate design to prospectively clarify the relationship between these variables and POHL may be an interesting direction for future study. Even so, the nomogram developed using existing data also performed well in all aspects. Third, compared with some previous studies, the mortality rate is a little higher in our results. This may be partly explained by the fact that a considerable portion of included patients underwent emergent cardiac surgery and aortic dissection surgery, which may significantly increase the overall mortality. The inclusion of multiple surgical 
types may help obtain a larger sample size, however, studies designed specific for each type of cardiac surgery may be more instructive for clinical practice.

\section{Conclusions}

To our knowledge, this is the first large-sized study reporting nomogram for POHL after cardiac surgery. Five preoperative and two intraoperative predictors were identified by multivariate logistic regression analysis and then a nomogram was constructed and validated. The nomogram performed well in discrimination, calibration and assessment of clinical usefulness. Four risk intervals were divided to better facilitate clinical practice. The nomogram may have clinical utility in risk evaluation, identification of high-risk population, and risk modification.

\section{Acknowledgments}

The authors sincerely thank Dr. Jehane Michael for his language help.

Funding: This work was supported by the National Natural Science Foundation of China (Grant No. 81800413).

\section{Footnote}

Reporting Checklist: The authors have completed the TRIPOD reporting checklist. Available at https://dx.doi. org/10.21037/jtd-21-1004

Data Sharing Statement: Available at https://dx.doi. org/10.21037/jtd-21-1004

Conflicts of Interest: All authors have completed the ICMJE uniform disclosure form (available at https://dx.doi. org/10.21037/jtd-21-1004). The authors have no conflicts of interest to declare.

Ethical Statement: The authors are accountable for all aspects of the work in ensuring that questions related to the accuracy or integrity of any part of the work are appropriately investigated and resolved. The study was conducted in accordance with the Declaration of Helsinki (as revised in 2013). The study was approved by the Ethics Committee of Tongji Medical College of Huazhong University of Science and Technology (IORG No. IORG0003571) and individual consent for this retrospective analysis was waived.
Open Access Statement: This is an Open Access article distributed in accordance with the Creative Commons Attribution-NonCommercial-NoDerivs 4.0 International License (CC BY-NC-ND 4.0), which permits the noncommercial replication and distribution of the article with the strict proviso that no changes or edits are made and the original work is properly cited (including links to both the formal publication through the relevant DOI and the license). See: https://creativecommons.org/licenses/by-nc-nd/4.0/.

\section{References}

1. Haas SA, Lange T, Saugel B, et al. Severe hyperlactatemia, lactate clearance and mortality in unselected critically ill patients. Intensive Care Med 2016;42:202-10.

2. Khosravani H, Shahpori R, Stelfox HT, et al. Occurrence and adverse effect on outcome of hyperlactatemia in the critically ill. Crit Care 2009;13:R90.

3. Hajjar LA, Almeida JP, Fukushima JT, et al. High lactate levels are predictors of major complications after cardiac surgery. J Thorac Cardiovasc Surg 2013;146:455-60.

4. Maillet JM, Le Besnerais P, Cantoni M, et al. Frequency, risk factors, and outcome of hyperlactatemia after cardiac surgery. Chest 2003;123:1361-6.

5. Evans AS, Levin MA, Lin HM, et al. Prognostic Value of Hyperlactatemia and Lactate Clearance After Mitral Valve Surgery. J Cardiothorac Vasc Anesth 2018;32:636-43.

6. Duchnowski P, Hryniewiecki T, Kuśmierczyk M, et al. The usefulness of perioperative lactate blood levels in patients undergoing heart valve surgery. Kardiochir Torakochirurgia Pol 2019;16:114-7.

7. Hoshino $\mathrm{Y}$, Kinoshita $\mathrm{O}$, Ono $\mathrm{M}$. The Incidence, Risk Factors, and Outcomes of Hyperlactatemia after Heart Transplantation. Int Heart J 2018;59:81-6.

8. Minton J, Sidebotham DA. Hyperlactatemia and Cardiac Surgery. J Extra Corpor Technol 2017;49:7-15.

9. Ranucci $M$, De Toffol B, Isgrò $G$, et al. Hyperlactatemia during cardiopulmonary bypass: determinants and impact on postoperative outcome. Crit Care 2006;10:R167.

10. Govender P, Tosh W, Burt C, et al. Evaluation of Increase in Intraoperative Lactate Level as a Predictor of Outcome in Adults After Cardiac Surgery. J Cardiothorac Vasc Anesth 2020;34:877-84.

11. O'Connor E, Fraser JF. The interpretation of perioperative lactate abnormalities in patients undergoing cardiac surgery. Anaesth Intensive Care 2012;40:598-603.

12. Abraham BP, Prodhan P, Jaquiss RD, et al. Cardiopulmonary bypass flow rate: a risk factor for 
hyperlactatemia after surgical repair of secundum atrial septal defect in children. J Thorac Cardiovasc Surg 2010;139:170-3.

13. Ranucci M, Carboni G, Cotza M, et al. Hemodilution on cardiopulmonary bypass as a determinant of early postoperative hyperlactatemia. PLoS One 2015;10:e0126939.

14. Demers P, Elkouri S, Martineau R, et al. Outcome with high blood lactate levels during cardiopulmonary bypass in adult cardiac operation. Ann Thorac Surg 2000;70:2082-6.

15. DeLong ER, DeLong DM, Clarke-Pearson DL. Comparing the areas under two or more correlated receiver operating characteristic curves: a nonparametric approach. Biometrics 1988;44:837-45.

16. Meregalli A, Oliveira RP, Friedman G. Occult hypoperfusion is associated with increased mortality in hemodynamically stable, high-risk, surgical patients. Crit Care 2004;8:R60-5.

17. Kogan A, Preisman S, Bar A, et al. The impact of hyperlactatemia on postoperative outcome after adult cardiac surgery. J Anesth 2012;26:174-8.

18. Worrell SG, Haug K, Dubovoy A, et al. Is Lactic Acidosis After Lung Transplantation Associated With Worse Outcomes? Ann Thorac Surg 2020;110:434-40.

19. Matteucci M, Ferrarese S, Cantore C, et al. Hyperlactatemia during cardiopulmonary bypass: risk factors and impact on surgical results with a focus on the long-term outcome. Perfusion 2020;35:756-62.

20. Raper RF, Cameron G, Walker D, et al. Type B lactic acidosis following cardiopulmonary bypass. Crit Care Med 1997;25:46-51.

21. Jackman L, Shetty N, Davies P, et al. Late-onset hyperlactataemia following paediatric cardiac surgery. Intensive Care Med 2009;35:537-45.

22. Cobianchi L, Peloso A, Filisetti C, et al. Serum lactate level as a useful predictor of clinical outcome after surgery: an unfulfilled potential? J Thorac Dis 2016;8:E295-7.

23. Jansen TC, van Bommel J, Schoonderbeek FJ, et al. Early lactate-guided therapy in intensive care unit patients: a multicenter, open-label, randomized controlled trial. Am J Respir Crit Care Med 2010;182:752-61.

24. Pojar M, Mand'ák J, Cibícek N, et al. Peripheral tissue metabolism during off-pump versus on-pump coronary artery bypass graft surgery: the microdialysis study. Eur J Cardiothorac Surg 2008;33:899-905.

25. Bahlmann L, Misfeld M, Klaus S, et al. Myocardial redox state during coronary artery bypass grafting assessed with microdialysis. Intensive Care Med 2004;30:889-94.
26. Gasparovic H, Plestina S, Sutlic Z, et al. Pulmonary lactate release following cardiopulmonary bypass. Eur J Cardiothorac Surg 2007;32:882-7.

27. Zakkar M, Guida G, Suleiman MS, et al. Cardiopulmonary bypass and oxidative stress. Oxid Med Cell Longev 2015;2015:189863.

28. Gutierrez G, Williams JD. The riddle of hyperlactatemia. Crit Care 2009;13:176.

29. Baikoussis NG, Papakonstantinou NA, Apostolakis E. The "benefits" of the mini-extracorporeal circulation in the minimal invasive cardiac surgery era. J Cardiol 2014;63:391-6.

30. Inoue S, Kuro M, Furuya H. What factors are associated with hyperlactatemia after cardiac surgery characterized by well-maintained oxygen delivery and a normal postoperative course? A retrospective study. Eur J Anaesthesiol 2001;18:576-84.

31. Koch CG, Li L, Sessler DI, et al. Duration of red-cell storage and complications after cardiac surgery. N Engl J Med 2008;358:1229-39.

32. Kopterides P, Theodorakopoulou M, Nikitas N, et al. Red blood cell transfusion affects microdialysis-assessed interstitial lactate/pyruvate ratio in critically ill patients with late sepsis. Intensive Care Med 2012;38:1843-50.

33. Koch CG, Li L, Duncan AI, et al. Morbidity and mortality risk associated with red blood cell and blood-component transfusion in isolated coronary artery bypass grafting. Crit Care Med 2006;34:1608-16.

34. Koch CG, Li L, Van Wagoner DR, et al. Red cell transfusion is associated with an increased risk for postoperative atrial fibrillation. Ann Thorac Surg 2006;82:1747-56.

35. Remy KE, Hall MW, Cholette J, et al. Mechanisms of red blood cell transfusion-related immunomodulation. Transfusion 2018;58:804-15.

36. Marik PE, Sibbald WJ. Effect of stored-blood transfusion on oxygen delivery in patients with sepsis. JAMA 1993;269:3024-9.

37. Duchnowski P, Hryniewiecki T, Kuśmierczyk M, et al. Red cell distribution width as a predictor of multiple organ dysfunction syndrome in patients undergoing heart valve surgery. Biol Open 2018;7:bio036251.

38. Duchnowski P, Hryniewiecki T, Kuśmierczyk M, et al. Performance of the EuroSCORE II and the Society of Thoracic Surgeons score in patients undergoing aortic valve replacement for aortic stenosis. J Thorac Dis 2019;11:2076-81.

39. Hensley NB, Gyi R, Zorrilla-Vaca A, et al. Retrograde 
Autologous Priming in Cardiac Surgery: Results From a Systematic Review and Meta-analysis. Anesth Analg 2021;132:100-7.

40. Shehata N, Mistry N, da Costa BR, et al. Restrictive compared with liberal red cell transfusion strategies in cardiac surgery: a meta-analysis. Eur Heart J 2019;40:1081-8.

41. Mueller MM, Van Remoortel H, Meybohm P, et al. Patient Blood Management: Recommendations From the 2018 Frankfurt Consensus Conference. JAMA 2019;321:983-97.

42. Litmathe J, Boeken U, Bohlen G, et al. Systemic inflammatory response syndrome after extracorporeal circulation: a predictive algorithm for the patient at risk. Hellenic J Cardiol 2011;52:493-500.

43. Dacey LJ, DeSimone J, Braxton JH, et al. Preoperative white blood cell count and mortality and morbidity after coronary artery bypass grafting. Ann Thorac Surg 2003;76:760-4.

44. Spencer S, Tang A, Khoshbin E. Leukodepletion for patients undergoing heart valve surgery. Cochrane Database Syst Rev 2013;(7):CD009507.

45. Khoshbin E, Spencer S, Solomon L, et al. Is there a renoprotective value to leukodepletion during heart valve surgery? A randomized controlled trial (ROLO). J

Cite this article as: Wang S, Wang D, Huang X, Wang H, Le S, Zhang J, Du X. Development and validation of a nomogram model for early postoperative hyperlactatemia in patients undergoing cardiac surgery. J Thorac Dis 2021;13(9):5395-5408. doi: $10.21037 /$ jtd-21-1004
Cardiothorac Surg 2021;16:58.

46. Sutton SW, Patel AN, Chase VA, et al. Clinical benefits of continuous leukocyte filtration during cardiopulmonary bypass in patients undergoing valvular repair or replacement. Perfusion 2005;20:21-9.

47. Andersen LW, Holmberg MJ, Doherty M, et al. Postoperative Lactate Levels and Hospital Length of Stay After Cardiac Surgery. J Cardiothorac Vasc Anesth 2015;29:1454-60.

48. Lazzeri C, Bonizzoli M, Cianchi G, et al. Lactate and Echocardiography Before Veno-Venous Extracorporeal Membrane Oxygenation Support. Heart Lung Circ 2018;27:99-103.

49. Mustafa I, Roth H, Hanafiah A, et al. Effect of cardiopulmonary bypass on lactate metabolism. Intensive Care Med 2003;29:1279-85.

50. Levraut J, Ciebiera JP, Chave S, et al. Mild hyperlactatemia in stable septic patients is due to impaired lactate clearance rather than overproduction. Am J Respir Crit Care Med 1998;157:1021-6.

51. Zymliński R, Biegus J, Sokolski M, et al. Increased blood lactate is prevalent and identifies poor prognosis in patients with acute heart failure without overt peripheral hypoperfusion. Eur J Heart Fail 2018;20:1011-8. 